\title{
THE USE OF A MOBILE ROBOT FOR COMPLETE SAMPLE MANAGEMENT IN A CELL CULTURE PILOT PLANT
}

\author{
Martin Wojtczyk ${ }^{1,2}$, Rüdiger Heidemann ${ }^{1}$, Klaus Joeris ${ }^{1}$, Chun Zhang ${ }^{1}$, \\ Mark Burnett ${ }^{1}$, Alois Knoll ${ }^{2}$ \& Konstantin Konstantinov ${ }^{1}$ \\ ${ }^{I}$ Bayer HealthCare, Biological Products, Process Sciences $R \& D$, \\ 800 Dwight Way, Berkeley, CA 94710, USA \\ ${ }^{2}$ Technical University of Munich, Department of Computer Sciences, \\ Robotics \& Embedded Systems, Boltzmannstr. 3, D-85748 Garching, Germany
}

\begin{abstract}
A mobile robot system was developed that is capable of automating the complete sample management in a biotechnological laboratory. The robot consists of a wheeled platform and a mounted industrial robot arm with a gripper tool attached to it. The proper interaction with the biotechnological devices is given by the use of a colour camera for object recognition, a force/torque sensor to prevent damages and laser scanners for localisation. Furthermore necessary changes to the environment of the robot are kept to a minimum. By providing a scripting language, the robot system can be easily adapted to new devices and further tasks. By the use of this autonomous robot operating distinct devices, a fully automated sample management system was established which is available day and night.
\end{abstract}

Key words: Automation, Cell Culture, Mobile Robot, Sample Management

\section{INTRODUCTION}

Sample management is an inevitable part during the development and production of biopharmaceuticals to keep track of the growth conditions for the optimisation of the harvest time during development and to ensure the repeatability during production. Monitoring and controlling the growth 
parameters for a cultivation usually causes a high human effort for weeks nights and weekends including.

For this reason an autonomous mobile robot was previously developed at the University of Bielefeld which is capable of automating the entire sample management in a cell culture pilot plant (Lütkemeyer et al. 2002, Scherer et al. 2003, Knoll et al. 2004, Scherer 2004 and Poggendorf 2004).

The robot's role in the sample management process is similar to a human's. After drawing a sample from a bioreactor utilising a sampling device, it is brought to a pipetting station which is capable of taking out a specific amount of aliquot and diluting it for examination at a Cedex cell counter. For archiving, the cells are separated from the sample in a centrifuge, tagged with barcodes, registered at an appropriate scanner and finally stored in a freezer. Meanwhile the process control system evaluates the received data and adjusts the production parameters if necessary, see Figure 1.

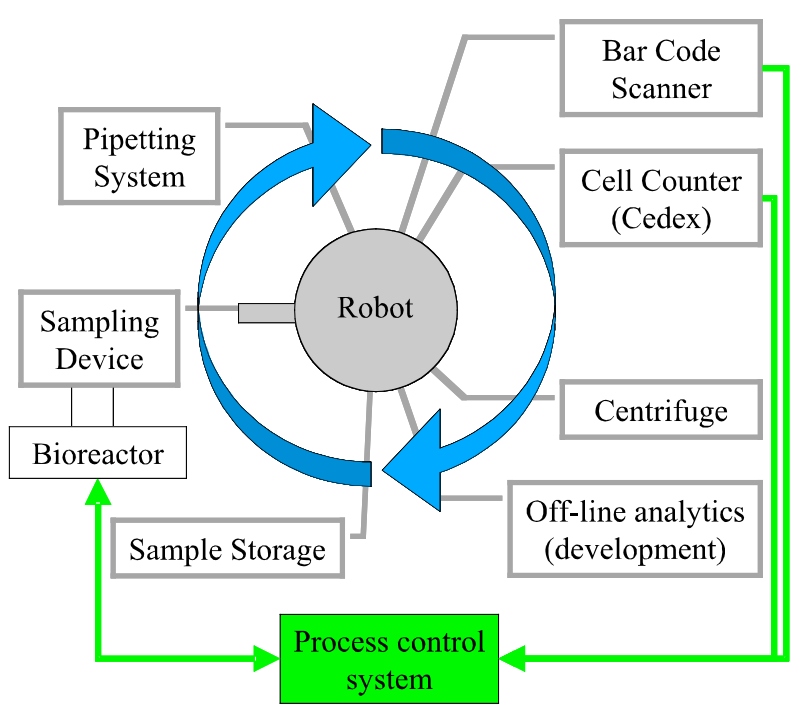

Figure 1. The automated sample management process.

\section{ROBOT}

A robot needs to accomplish certain requirements, to be used for sample management in an environment which is made for humans. Of course it needs to reach the distinct devices, to identify them and to be able to use 
them with care, not to damage anything. Furthermore it is supposed to handle several sizes of tubes.

\subsection{Hardware setup}

On this account, a battery driven mobile platform MP-L655 from Neobotix was chosen to provide the robot with the necessary mobility and autonomy, being controlled by its own platform computer running a Linux operating system. Equipped with an industrial seven joints robot arm PA-10 from Mitsubishi Heavy Industries and a two-fingered gripper tool attached to it, it is able to reach and to operate the involved devices and to carry the desired tubes, see Figure 2.

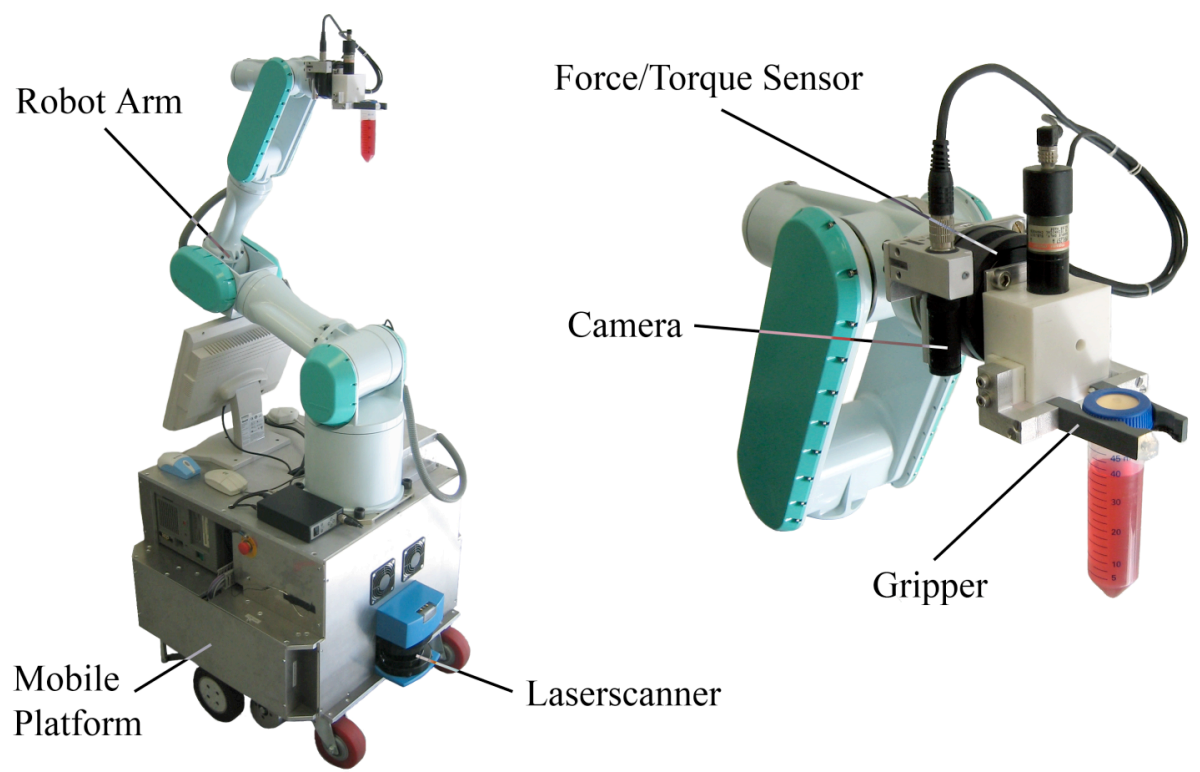

Figure 2. Mobile robot platform and close-up of the robot tool carrying a 50ml tube.

\subsection{Localisation and Navigation}

Two $180^{\circ}$ laser scanners at the front and back side of the platform detect laser scan markers attached to the walls of a biotechnical laboratory whose positions are matched against the known entries in a map. This method enables the robot to estimate its position and orientation up to 38 times per second, see Figure 3. In conjunction with the given map the $\mathrm{A}^{*}$ algorithm and tangent graph are applied to find the shortest non colliding way through the lab to the goal positions. If dynamic obstacles - like for example human 
personnel - are detected to be too close to the robot on its way to its target position, it stops immediately until the obstacle is out of scope again.

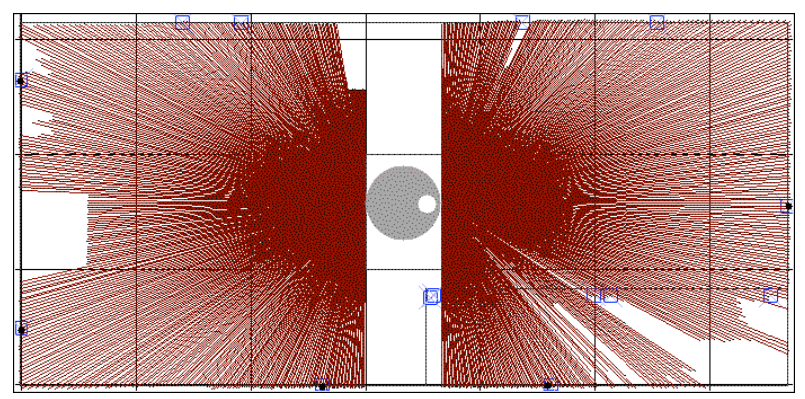

Figure 3. Localisation with the aid of laser scanners. The robot's environment seen from the top at the height of the laser scanners showing the acquired distance information.

\subsection{Device Interaction}

Because of the need for high accuracy while operating the laboratory devices, the robot tool is supplied with additional sensors. Since cameras provide a very natural and comprehensive sensor input, a camera is included at the gripper tool to investigate the device, the robot is working at. Thus it is possible to compensate little navigation errors caused by noise during the laser scans and to guide the robot arm with an accuracy of one millimetre.

Image processing often is time consuming, however it can be accelerated if unnecessary information is filtered out. For the current object recognition a colour based region growing approach was chosen evaluating YUV images. The algorithm looks for compound segments of the colours blue, red and yellow and calculates their centres of gravity to match their relative position between each other against entries in the robot's database which makes object identification possible, where objects contain enough unique colour information or at least are supplied with appropriate colour markers, see Figure 4. The absolute position of the centres of gravity supply the necessary information for fine positioning the robot arm.

Once the position and orientation of the current device or object is calculated, a scripted action is triggered which may be supplied with force constraints guarded by the force/torque sensor not to cause harm to the equipment or even to human personnel. By the use of the general colour based identification method and the scripting language, the robot can easily be adapted to new devices. 

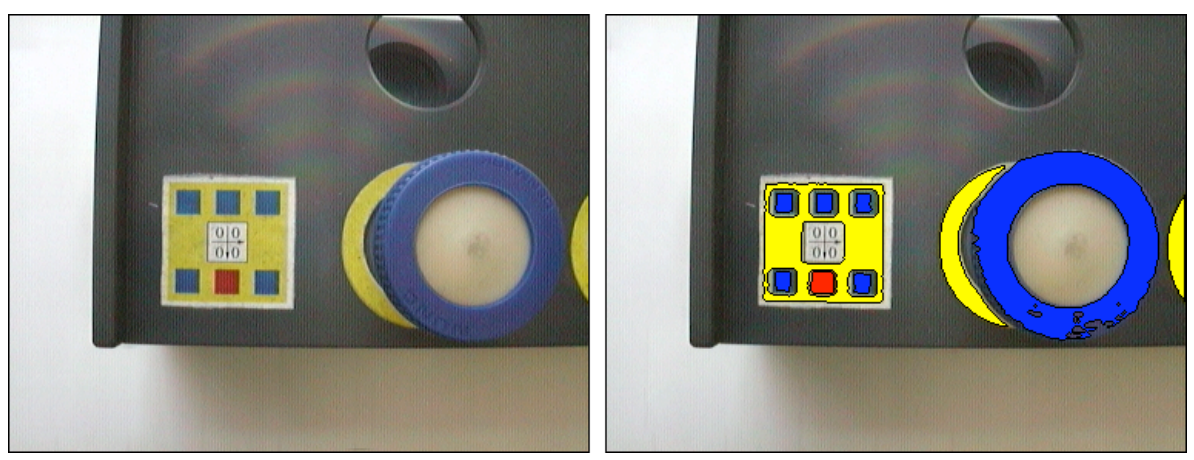

Figure 4. Image processing for object recognition. On the left the captured image of an optical marker and a $50 \mathrm{ml}$ tube, on the right the processed image showing the different segments for the search colours.

\section{CONCLUSION}

The goal of the project is to combine research results in both key technologies Robotics and Biotechnology. After the successful utilisation in a cell culture pilot plant in Germany the robot was recently transferred to Bayer HealthCare in Berkeley, USA, to be adjusted to the environment and to new Bayer-specific equipment like an automated sampling valve and a LabView based supervisory system for later use in development and production of biopharmaceuticals.

\section{REFERENCES}

Lütkemeyer, D., Poggendorf, I., Scherer, T., Zhang, J., Knoll, A., and Lehmann, J., 2000, First steps in robot automation of sampling and sample management during cultivation of mammalian cells in pilot scale. Biotechnol. Prog., 16: 822-828

Scherer, T., Poggendorf, I., Schneider, A., Westhoff, D., Zhang, J., Lütkemeyer, D., Lehmann, J., and Knoll, A, 2003, A service robot for automating the sample management in biotechnological cell cultivations. Proceedings of the 30th Annual Conference of Computers in Cardiology, Lisbon, Portugal

Knoll, A., Scherer, T., Poggendorf, I., Lütkemeyer, D., and Lehmann, J., 2004, Flexible automation of cell culture and tissue engineering tasks. Biotechnol. Prog. 20: 1825-1835

Scherer, T., 2004, A Mobile Service Robot for Automisation of Sample Taking and Sample Management in a Biotechnological Pilot Laboratory. PhD thesis, University of Bielefeld

Poggendorf, I., 2004, Einsatz eines Serviceroboters zur Automatisierung der Probenentnahme und des Probenmanagements während Kultivierungen tierischer Zellen in einer Technikumsumgebung. [online] PhD thesis, University of Bielefeld. Available from: http://nbn-resolving.de/urn/resolver.pl?urn=urn:nbn:de:hbz:361-5651 